\title{
Editorial
}

\section{Functionalized Nanocomposites for Environmental Applications}

\author{
Tifeng Jiao, ${ }^{1}$ Lavinia Balan, ${ }^{2}$ Xinqing Chen, ${ }^{3}$ and Qingrui Zhang ${ }^{4}$ \\ ${ }^{1}$ School of Environmental and Chemical Engineering, Yanshan University, Qinhuangdao 066004, China \\ ${ }^{2}$ Institute of Materials Science of Mulhouse (IS2M), 15 Rue Jean Starcky, P.O. Box 2488, 68057 Mulhouse Cedex, France \\ ${ }^{3}$ Department of Chemical and Biomolecular Engineering, Hong Kong University of Science and Technology, Clear Water Bay, \\ Kowloon, Hong Kong \\ ${ }^{4}$ Department of Environmental Engineering, Yanshan University, Qinhuangdao 066004, China
}

Correspondence should be addressed to Tifeng Jiao; tfjiao@ysu.edu.cn

Received 4 March 2014; Accepted 4 March 2014; Published 26 March 2014

Copyright (C) 2014 Tifeng Jiao et al. This is an open access article distributed under the Creative Commons Attribution License, which permits unrestricted use, distribution, and reproduction in any medium, provided the original work is properly cited.

During the last decades, using functionalized nanocomposites with nanoscale precision represents one of the most important bottom-up approaches for nanotechnology and has become a major issue in the field of environmental chemistry. The chemical functionalization and self-assemblies of nanocomposites and subsequent environmental applications of well-defined micro-/nanostructures have multiple important impacts upon various areas. In this sense, the sophisticated nanocomposites with multiple components have deepened the insight of nanomaterials and enriched the content of environmental chemistry.

This special issue addresses the research studies on the functionalized process and environmental applications of nanocomposites. It contains eleven selected research articles. Z. Xu et al. prepared the adsorption performance of monobutyl phthalate onto macroporous base anionexchange resins. $H$. Zhou et al. reported a new absorbent of Fe loaded biomass char and investigated the enhanced adsorption capacity for phosphate in water. J. Luo et al. prepared the synthesis of mesoporous Fe contained g- $\mathrm{C}_{3} \mathrm{~N}_{4}$ (m-Fe- $\mathrm{C}_{3} \mathrm{~N}_{4}$ ) photocatalyst by using $\mathrm{SiO}_{2}$ nanoparticles as hard template and dicyandiamide as precursor and investigated the photocatalytic activity of photodegradation of Rhodamine. B. S. Wang et al. reported the preparation of iron-doped titania thin film by means of sol-gel method to degrade typical indoor air pollutants under daylight illumination. L. Wang et al. reported the use of living spirulina as novel photosensitizer to construct spirulina biosolar cell. T. Jiao et al. prepared the design of some luminol imide derivatives with different substituent groups and investigated the electrochemiluminescent detection of hydrogen peroxide with a view of developing new biosensors. F. Jin reported the preparation of glucan- $\mathrm{Fe}_{3} \mathrm{O}_{4}$ magnetic nanoparticles and in vivo distribution in mice. L. Wang et al. prepared modified expanded graphite samples and measured the sorption capacity for some oils. W. Guo et al. prepared $\mathrm{Bi}_{2} \mathrm{MoO}_{6}$ single-crystallites by hydrothermal method in the presence of different surfactants and measured the photocatalytic activities for the degradation of methylene blue. Y. Qiao et al. demonstrated the investigation on the structure and electrochemical characteristics of La-Ce-Mg-Al-Ni hydrogen storage alloy. S. Wang et al. reported the preparation and magnetic properties of anisotropic $(\mathrm{Sm}, \mathrm{Pr}) \mathrm{Co}_{5} / \mathrm{Fe}$ nanocomposites particles by electroless plating. The effect of Fe content on the magnetic properties of the magnets is also discussed.

Altogether, the guest editors would like to express sincere appreciation to all the authors for their contribution. Moreover, thanks are extended to all reviewers for their time spent on enhancing the quality of these papers.

Tifeng Jiao

Lavinia Balan

Xinqing Chen

Qingrui Zhang 

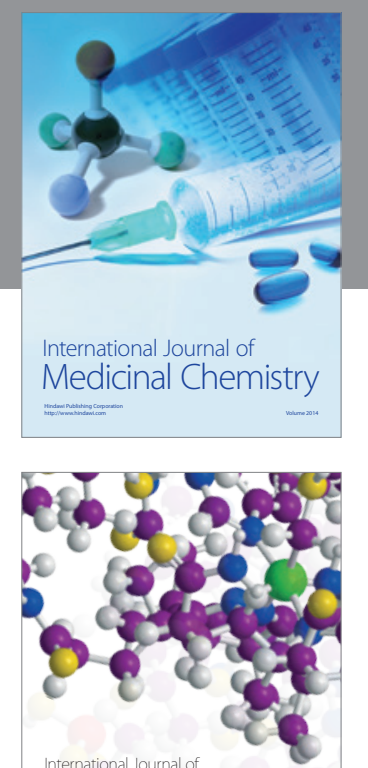

\section{Carbohydrate} Chemistry

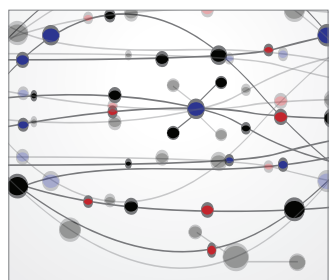

The Scientific World Journal
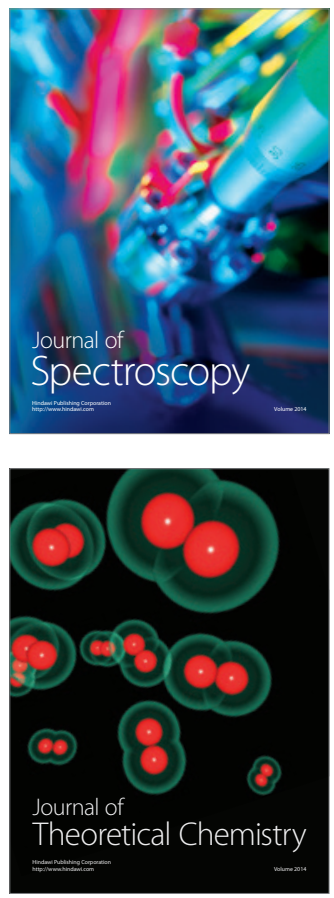
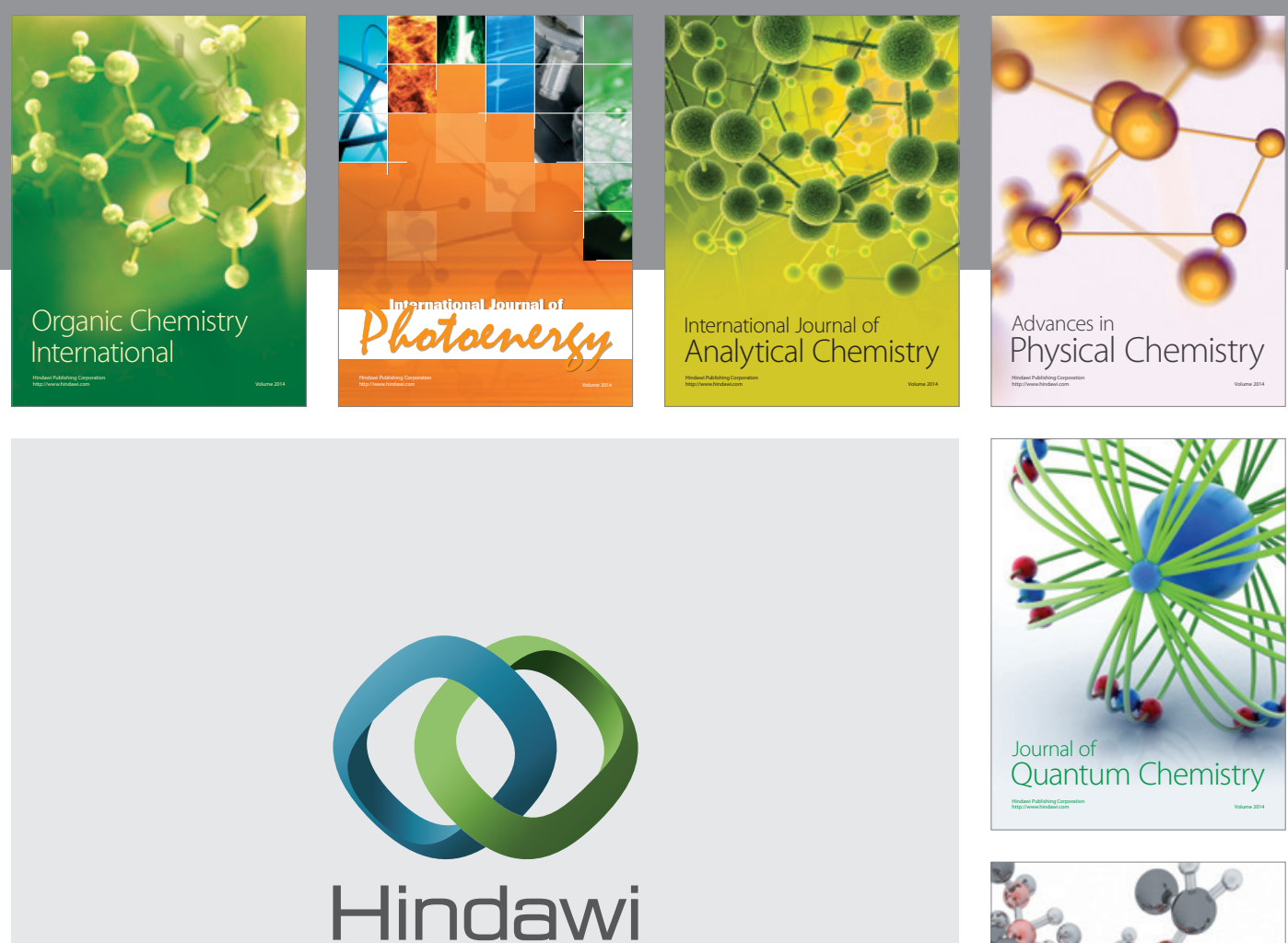

Submit your manuscripts at

http://www.hindawi.com

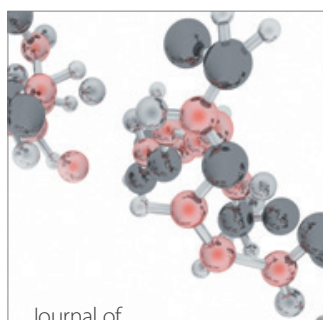

Analytical Methods

in Chemistry

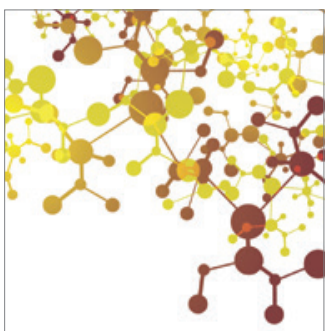

Journal of

Applied Chemistry

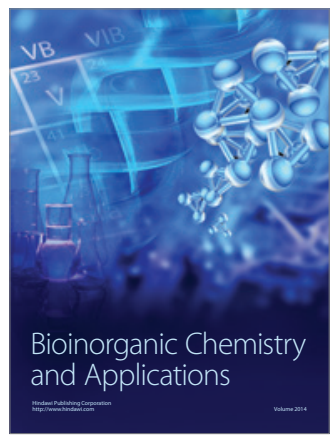

Inorganic Chemistry
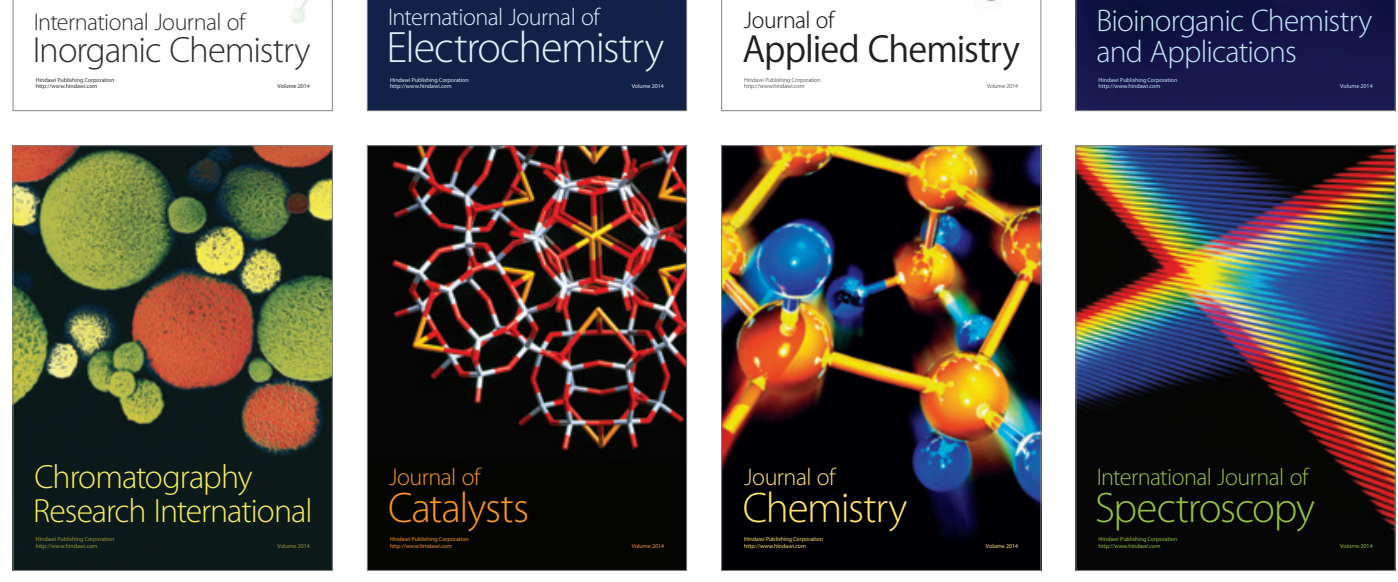